\title{
Thin Structure Segmentation and Visualization in Three-Dimensional Biomedical Images: A Shape-Based Approach
}

\author{
Adam Huang, Gregory M. Nielson, Senior Member, IEEE, Anshuman Razdan, Member, IEEE, \\ Gerald E. Farin, D. Page Baluch, and David G. Capco
}

\begin{abstract}
This paper presents a shape-based approach in extracting thin structures, such as lines and sheets, from threedimensional (3D) biomedical images. Of particular interest is the capability to recover cellular structures, such as microtubule spindle fibers and plasma membranes, from laser scanning confocal microscopic (LSCM) data. Hessian-based shape methods are reviewed. A synthesized linear structure is used to evaluate the sensitivity of the multiscale filtering approach in extracting closely positioned fibers. We find that the multiscale approach tends to fuse lines together, which makes it unsuitable for visualizing mouse egg spindle fibers. Single-scale Gaussian filters, balanced between sensitivity and noise resistance, are adopted instead. In addition, through an ellipsoidal Gaussian model, the eigenvalues of the Hessian matrix are quantitatively associated with the standard deviations of the Gaussian model. Existing shape filters are simplified and applied to LSCM data. A significant improvement in extracting closely positioned thin lines is demonstrated by the resultant images. Further, the direct association of shape models and eigenvalues makes the processed images more understandable qualitatively and quantitatively.
\end{abstract}

Index Terms-Angiography, biomedical image processing, Hessian matrix, image enhancement, laser scanning confocal microscopy, multiscale filtering, segmentation, visualization.

\section{INTRODUCTION}

$\mathrm{T}$ HIN structures such as lines and sheets are important features in many biological and medical imaging applications. Medical examples include blood vessels in angiography, bronchi in chest computed tomography (CT) scan, and cortices in brain magnetic resonance imaging (MRI) scan. Visualization of such structures provides crucial information in planning and navigation during interventional therapy and biopsy as well as for diagnostic purposes. Biological examples include plasma membranes and cellular scaffolds [2] in confocal microscopy data. Visualization of their spatial arrangement and temporal transformations can help biologists to study cellular organelles and mechanisms. There has been a considerable amount of work done on the enhancement and extraction of blood vessels. It should be a straightforward task to apply existing blood vessel extraction algorithms to similar line

- A. Huang is with the Diagnostic Radiology Department, Warren Grant Magnuson Clinical Center, National Institutes of Health, Building 10, Room 1C671, 10 Center Drive MSC 1182, Bethesda, MD 20892-1182. E-mail: hhuang@cc.nih.gov.

- G.M. Nielson and G.E. Farin are with the Department of Computer Science and Engineering, Ira A. Fulton School of Engineering, Arizona State University, Tempe, AZ 85287. E-mail: \{nielson, farin\}@asu.edu.

- A. Razdan is with the Partnership for Research In Stereo Modeling (PRISM), PO Box 878609, Arizona State University, Tempe, AZ 85287. E-mail:razdan@asu.edu.

- D.P. Baluch and D.G. Capco are with the Department of Biology, School of Life Sciences, Arizona State University, Tempe, AZ 85287.

E-mail: \{page.baluch, dcapco\}@asu.edu.

Manuscript received 24 Aug. 2004; revised 2 Feb. 2005; accepted 19 May 2005; published online 9 Nov. 2005.

For information on obtaining reprints of this article, please send e-mail to: tvcg@computer.org, and reference IEEECS Log Number TVCG-0094-0804. structures such as microtubule spindle fibers. However, biological images in the microscopic scale are much noisier and remain a challenge to the use of existing methods.

Fig. 1 demonstrates the challenge posed by biological data. Fig. 1a is a volume rendering [6], [19], [20] image of the meiotic microtubule spindle fibers of a mouse egg data set obtained from a laser scanning confocal microscope (LSCM) [12], [25], [31]. The bundle of spindle fibers appears as a barrel-shaped structure which has distinguishable lines in the central region and fused blobs at each end. Fig. $1 \mathrm{~b}$ is an isosurface extraction of the fibers from the same original data set rendered by the marching cubes (MC) algorithm [21], [24]. As the intensity values of spindle fibers detected by an LSCM vary greatly, without proper image processing and enhancement, volume rendering barely provides a vague $3 \mathrm{D}$ line image, while isosurface rendering cannot even depict the targeted structures properly. In order to recover the fibers buried in a noisy background, a shapebased multiscale line filtering method, as suggested by Sato et al. in [26] and [27], is applied to enhance the spindle fibers. The extracted isosurfaces of the filtered fibers are shown in Fig. 1c. Although line structures in Fig. 1c are more visible compared to Fig. $1 \mathrm{~b}$, a great portion of anticipated fibers are still fused and indistinguishable near both poles.

This paper reviews shape-related work and examines the Hessian-based multiscale filtering approach in extracting thin structures, such as lines and sheets, from 3D biomedical images. Of particular interest is the capability to recover cellular structures such as microtubule spindle fibers and plasma membranes from 3D confocal microscopy data. A synthesized linear structure, which mimics spindle 


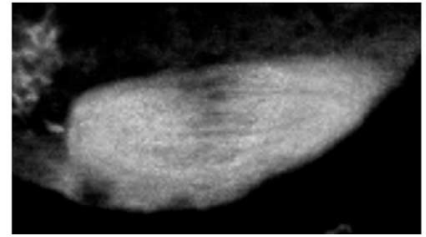

(a)

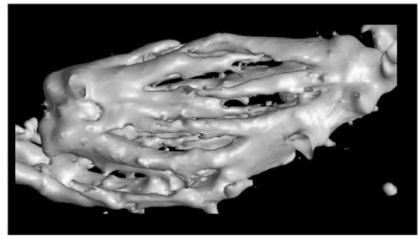

(c)

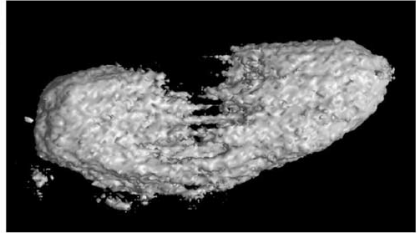

(b)

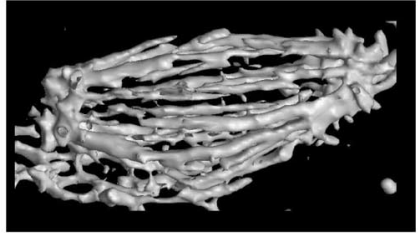

(d)
Fig. 1. Meiotic microtubule spindle fibers of a mouse egg. (a) Volume rendering of the spindle fibers. (b) Surface rendering of the original data. (c) Surface rendering of the spindle fibers enhanced by multiscale line filtering. (d) Surface rendering of the spindle fibers extracted by applying our new method.

fibers, is used to evaluate the performance of a multiscale line filtering implementation suggested in [27] in extracting closely placed fibers. We find that the multiscale approach tends to fuse lines together, which makes it unsuitable for mouse egg spindle fiber segmentation and visualization. Based on the evaluation, single-scale Gaussian filters, balanced between sensitivity and noise resistance, are adopted for mouse egg spindle fiber segmentation and visualization where multiscale filtering falls short. In addition, through an ellipsoidal Gaussian function model, the eigenvalues of the Hessian matrix are quantitatively associated with the standard deviations of the Gaussian model. Based on the analytically derived information, existing shape filters are simplified. Sheet, line, and blob structures are defined by specific, negative eigenvalues and segmented by thresholding. Fig. 1d depicts a much improved image of the extracted spindle fibers by using the proposed enhancement/segmentation methods. More spindle fibers are distinguishable from one end to the other. The proposed methods are tested on synthetic, LSCM, and angiography data. A significant improvement in extracting closely positioned thin lines is demonstrated by the resultant images. Further, the direct association of shape models and eigenvalues makes the processed images more understandable qualitatively and quantitatively.

\section{Shape-Related Work}

There are at least two viewpoints for "shape" in the context of image segmentation and visualization. The first viewpoint is in the forms of curves and surfaces. It is an intuitive human reflection, as Farin describes in [8], that the appearance of an object is judged by the flow of its curves. In this definition, lines have no width and surfaces have no thickness. This viewpoint corresponds to the boundary output from the image segmentation process. Based on this viewpoint and definition, surface models have been developed to construct computerized shape descriptions for geometric objects from a variety of measurements. The marching cubes [21], [24] algorithm and surface reconstruction from cross sections [4], [18] for volume data, point cloud fitting [7], [11], [14] for range images, and active deformable surfaces [17], [22], [23] for both range images and volume data are just some examples. In addition to surface reconstruction, shape-related characteristics are also applied to the realms of archeology and computer-aided analysis and diagnosis. Curvature, a local geometric property for shape description, is used as an index to analyze and categorize curves and surfaces of archeology artifacts [28]. It is also applied to brain cortex folding study [29] and automatic colonic polyp detection [16], [30], [32].

The second viewpoint of shape corresponds to the output from the image segmentation process in the forms of sets of data elements. In this viewpoint, curves have width and surfaces have thickness because they represent the internal regions which are enclosed by the curves or surfaces themselves. Take font design, for example. The shape of the boundary curves of a letter "a" is the main feature in the design process. However, it is the line width that makes a bold-faced letter "a" appear as different from a regular " $a$ " to the human eye. In many medical applications, the shape features such as the width and thickness of line and sheet structures are equally important as curve and surface descriptions. The thickness and density changes in the white and gray matter detected in brain MRI scans can provide physical evidence to diagnose the onset of early Alzheimer's disease or the effectiveness of clinical drug trials. The structures of blood vessels have measurable abnormalities in diameter such as stenoses and aneurysms. Such abnormalities reveal further information regarding the state of various diseases. For these diagnostic reasons, blood vessel detection and segmentation methods have been developed for years. Among many research efforts, shaperelated methods stand out as some of the most successful and proven approaches.

As observed in Fig. 2a, the blood vessels in retinal angiography typically appear as a tree-like pattern. Such a pattern consists of piecewise linear segments with a Gaussian-like profile. This means that the cross section of a blood vessel in a grayscale image appears as a Gaussian distribution function (see Figs. $2 b$ and $2 c$, for example). Chaudhuri et al. [5] illustrate three distinctive shape characteristics of blood vessels in retinal images: 1) Blood vessels usually have small curvatures and can be approximated by piecewise linear segments. 2) The grayscale profile along the direction perpendicular to a blood vessel as shown in Fig. 2c looks like a Gaussian function. 3) The width of a vessel is relatively constant within a range of a few (2 to 10) pixels.

A precise shape model for blood vessels in angiography is difficult to develop because of variations in tissue characteristics, light sources, and imaging systems' optical and electronic components. However, an approximation that conforms to principal features while maintaining calculation simplicity will be sufficient and acceptable for practical applications. The Gaussian vessel profile is a very popular model. It is generally described by a function similar to 


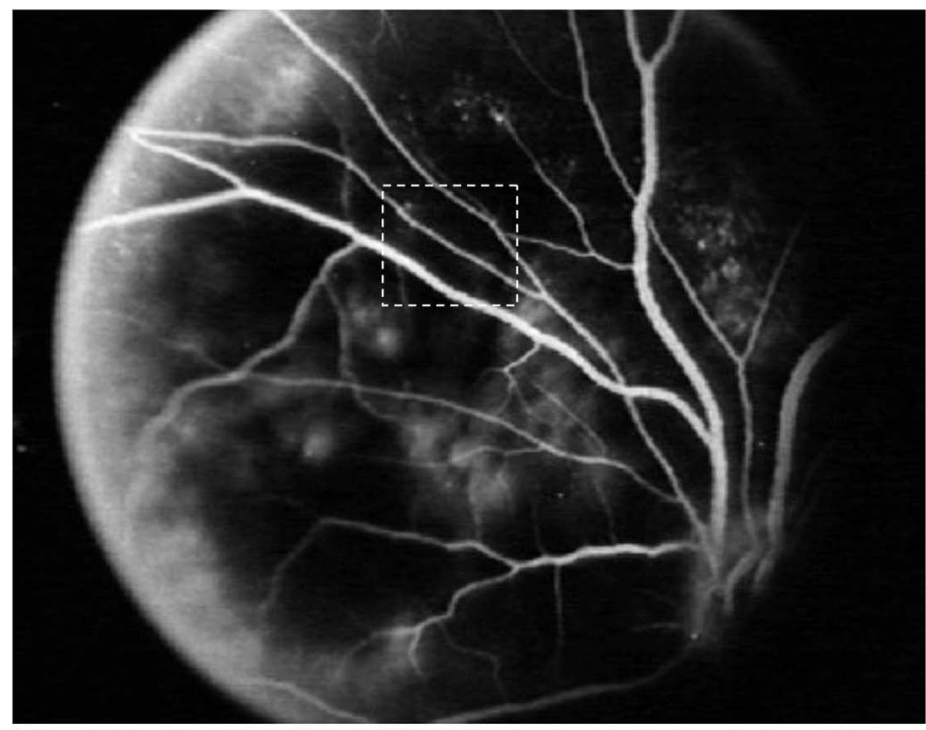

(a)

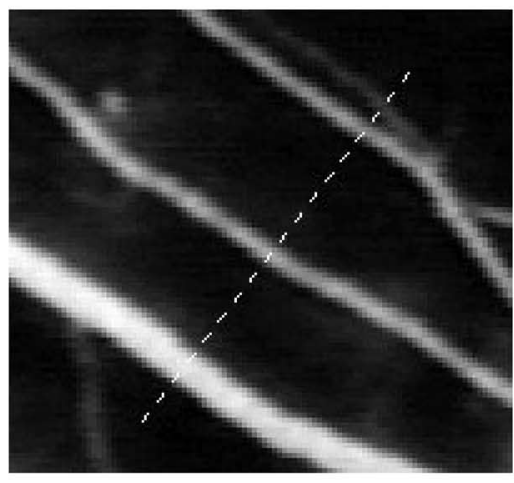

(b)

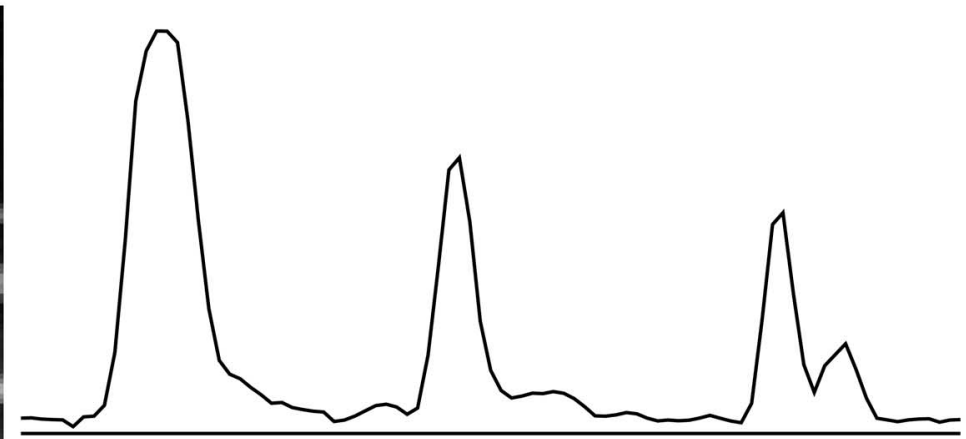

(c)

Fig. 2. (a) Fluorescein angiogram obtained from a fundus camera. (b) The magnified image of the bounded region. (c) The grayscale profile of a blood vessel sampled along the dotted line shown in (b).

$$
F(x, y)=A\left\{1+k \exp \left(-\frac{d^{2}}{2 \sigma^{2}}\right)\right\},
$$

where $d$ is the perpendicular distance between the point $(x, y)$ and the axis of the blood vessel, $\sigma$ is the standard deviation, $A$ is the local background intensity, and $k$ is a measure of the relative intensity between the vessel and its neighborhood. Gang et al. provide a statistical evaluation in [10] to prove the fitness of estimating vessel profiles with Gaussian functions. A comparison of various shape-related methods applied to retinal blood vessel images is given in [15].

For 3D angiography, Sato et al. [26], [27] propose shapeselective filters based on the eigenvalues of the 3D Hessian

$$
\mathbf{H}=\left[\begin{array}{lll}
F_{x x} & F_{x y} & F_{x z} \\
F_{y x} & F_{y y} & F_{y z} \\
F_{z x} & F_{z y} & F_{z z}
\end{array}\right],
$$

where the second partial derivatives of a volume intensity function $F(x, y, z)$ are represented as $F_{x x}=\frac{\partial^{2}}{\partial x^{2}} F, F_{y z}=\frac{\partial^{2}}{\partial y \partial z} F$, and so on. The Hessian matrix describes the second-order structure of local intensity variations in the neighborhood of each point of the intensity function $F$. Let $\lambda_{1}, \lambda_{2}$, and $\lambda_{3}$

$\left(\lambda_{1} \geq \lambda_{2} \geq \lambda_{3}\right)$ be the eigenvalues of $\mathbf{H}$ and $\mathbf{e}_{1}, \mathbf{e}_{2}, \mathbf{e}_{3}$ their corresponding eigenvectors, respectively. The eigenvector $\mathbf{e}_{1}$ represents the direction along which $F$ has the maximum directional second-derivative value $\lambda_{1}$. Similarly, along the direction $\mathbf{e}_{3}, F$ has the minimum directional secondderivative value $\lambda_{3}$. The eigenvector $\mathbf{e}_{2}$ is orthogonal to $\mathbf{e}_{1}$ and $\mathbf{e}_{3} ; \lambda_{2}$ is the corresponding directional second-derivative value. Assuming the targeted structures are brightened in contrast to the background, the conditions for categorizing local structures are summarized in Table 1.

Sato et al. propose sheet, line, and blob filters as

$$
\begin{gathered}
S_{\text {sheet }}= \begin{cases}\left|\lambda_{3}\right| \cdot \omega\left(\lambda_{2} ; \lambda_{3}\right) \cdot \omega\left(\lambda_{1} ; \lambda_{3}\right) & \lambda_{3}<0 \\
0, & \text { otherwise, }\end{cases} \\
S_{\text {line }}= \begin{cases}\left|\lambda_{3}\right| \cdot \psi\left(\lambda_{2} ; \lambda_{3}\right) \cdot \omega\left(\lambda_{1} ; \lambda_{2}\right) & \lambda_{3} \leq \lambda_{2}<0 \\
0, & \text { otherwise, }\end{cases} \\
S_{\text {blob }}= \begin{cases}\left|\lambda_{3}\right| \cdot \psi\left(\lambda_{2} ; \lambda_{3}\right) \cdot \psi\left(\lambda_{1} ; \lambda_{2}\right) & \lambda_{3} \leq \lambda_{2} \leq \lambda_{1}<0 \\
0, & \text { otherwise. }\end{cases}
\end{gathered}
$$


TABLE 1

Basic Conditions for Local Structures

\begin{tabular}{|l|l|l|}
\hline $\begin{array}{l}\text { Local } \\
\text { Structure }\end{array}$ & $\begin{array}{l}\text { Eigenvalue } \\
\text { Conditions }\end{array}$ & Examples \\
\hline sheet & $\lambda_{3}<<\lambda_{2} \cong \lambda_{1} \cong 0$ & $\begin{array}{l}\text { cortex, } \\
\text { membrane }\end{array}$ \\
\hline line & $\lambda_{3} \cong \lambda_{2}<<\lambda_{1} \cong 0$ & vessel, bronchus \\
\hline blob & $\lambda_{3} \cong \lambda_{2} \cong \lambda_{1}<<0$ & nodule \\
\hline
\end{tabular}

$\psi$ and $\omega$ are weight functions written as

$$
\psi\left(\lambda_{s} ; \lambda_{t}\right)= \begin{cases}\left(\lambda_{s} / \lambda_{t}\right)^{\gamma} & \lambda_{\mathrm{t}} \leq \lambda_{s}<0 \\ 0, & \text { otherwise }\end{cases}
$$

and

$$
\omega\left(\lambda_{s} ; \lambda_{t}\right)= \begin{cases}\left(1+\lambda_{s} /\left|\lambda_{t}\right|\right)^{\gamma} & \lambda_{\mathrm{t}} \leq \lambda_{s} \leq 0 \\ \left(1-\alpha \lambda_{s} /\left|\lambda_{t}\right|\right)^{\gamma} & \left|\lambda_{\mathrm{t}}\right| / \alpha>\lambda_{\mathrm{s}}>0 \\ 0, & \text { otherwise, }\end{cases}
$$

where $\gamma$ controls the sharpness of selectivity for the conditions of each local structure and $0<\alpha \leq 1$, which makes $\omega$ asymmetric. They suggest $\alpha=0.25$ and $\gamma=0.5$ (or 1) for typical applications. Extensive analysis of the measurement, including the effects of parameters $\alpha$ and $\gamma$, can be found in [27].

Frangi et al. apply a similar approach to both 2D and 3D vessel images with a modified line filter in [9]. The line filtering with the eigenvalues of the Hessian presented in [9], [26], [27] all takes a multiscale approach to detect vessels over a large size range. In this framework, differentiation is defined as a normalized convolution with derivatives of Gaussians:

$$
\begin{aligned}
& F_{x^{i} y^{j} z^{k}}\left(x, y, z ; \sigma_{f}\right) \\
&=\left\{\sigma_{f}^{i+j+k} \frac{\partial^{i+j+k}}{\partial x^{i} \partial y^{j} \partial z^{k}} G\left(x, y, z ; \sigma_{f}\right)\right\} * F(x, y, z) \\
&=\sigma_{f}^{i+j+k} \frac{d^{i}}{d x^{i}} G\left(x ; \sigma_{f}\right) * \\
&\left\{\frac{d^{j}}{d y^{j}} G\left(y ; \sigma_{f}\right) *\left\{\frac{d^{k}}{d z^{k}} G\left(z ; \sigma_{f}\right) * F(x, y, z)\right\}\right\},
\end{aligned}
$$

where $G$ is an isotropic 3D Gaussian function with standard deviation $\sigma_{f}$ and $\sigma_{f}^{i+j+k}$ the normalization factor. Let the filter response obtained from the normalized derivatives (8) represented by

$$
S_{\xi}\left(\sigma_{f}\right)
$$

where $\xi \in\{$ sheet, line, blob $\}$. The diameter of vessels can be determined by the $\sigma_{f}$ which generates a maximum response.

Besides eigenvalues, the eigenvectors of the Hessian provide local orientation information which is adopted by various tracing algorithms [1], [33], [34] for curvilinear object recovery. Alyward et al. [1] apply both the eigenvalues and eigenvectors of the Hessian in extracting tubular structures as intensity ridges. The width information is then obtained through multiscale responses from normalized convolutions.

Recently, a new technique, called diffusion tensor magnetic resonance imaging (DT-MRI) [3], has been found able to measure the anisotropic diffusion properties of biological tissues for classification. Diffusion tensor, which is represented as

$$
\mathbf{D}=\left[\begin{array}{ccc}
D_{x x} & D_{x y} & D_{x z} \\
D_{y x} & D_{y y} & D_{y z} \\
D_{z x} & D_{z y} & D_{z z}
\end{array}\right]
$$

has a notation similar to the Hessian. However, its elements have different physical meanings. Its diagonal terms represent correlations between molecular displacements in the same directions, where its off-diagonal terms reflect correlations between molecular displacements in orthogonal directions. In the case of an elongated cell, the tissue will have a preferred diffusion direction along the primary axis of the cell. Similar to the Hessian analysis, the preferred direction and diffusion can be derived from the eigenvalues and eigenvectors of D. Zhukov and Barr apply a fiber tracing method to recover and visualize the structure of the heart muscle fibers in [33] and white matter fibers in [34]. Differing from Gaussian convolution schemes, they compute weighted averages of tensor fields by using a least squares fitting to low polynomials with dynamic adjusted weights. The weights are determined based on directional information (eigenvectors), shape (eigenvalues), and intensity value.

In this paper, we present work on thin structure modeling using an ellipsoidal Gaussian function. We find that the eigenvalues of the Hessian are quantitatively associated with the standard deviations. Based on quantitative information, existing shape filter definitions are simplified. Regarding numerical differentiation, the multiscale approach, which tends to fuse closely positioned fibers in LSCM data, is replaced by single-scale filtering. Although good performance for low-resolution, noisy data such as DT-MRI is reported in [33], we keep our approach simple for two reasons. First, the complexity in selecting various parameters in [33] is high and the level of applicability to different image modules (such as LSCM) is unknown. Second, part of our project goal is to discover unknown cellular organelles. Therefore, we choose simple algorithms such as enhancement/thresholding type of edge detectors over more sophisticated line tracing methods to prevent introducing artifacts.

This paper is derived from [13] with additional work on ellipsoidal shape modeling and multiscale filtering evaluation. The derivation of simplified shape structure extraction methods from an ellipsoidal model is delineated in Section 3.1. The line filtering performance is evaluated in Section 3.2. Last, experimental results and conclusions are presented in Sections 4 and 5.

\section{Methods}

\subsection{Ellipsoidal Model}

A sheet, line, or blob structure summarized in Table 1 can be modeled by an ellipsoidal Gaussian function

$$
\begin{aligned}
F(x, y, z) & =\exp \left(-\frac{x^{2}}{2 \sigma_{x}^{2}}-\frac{y^{2}}{2 \sigma_{y}^{2}}-\frac{z^{2}}{2 \sigma_{z}^{2}}\right)+C+N(x, y, z) \\
& =F_{\text {ellipsoid }}+C+N(x, y, z)
\end{aligned}
$$

where $C$ is the constant noise term and $N(x, y, z)$ represents the nonconstant noise. With proper choices of standard deviations $\sigma_{x}, \sigma_{y}, \sigma_{z}$, and coordinate system rearrangement, a local sheet $\left(\sigma_{x}=1, \sigma_{y} \cong \sigma_{z}>>0\right)$, line $\left(\sigma_{x} \cong \sigma_{y} \cong 1\right.$, 
$\left.\sigma_{z}>>0\right)$, or blob $\left(\sigma_{x} \cong \sigma_{y} \cong \sigma_{z} \cong 1\right)$ structure can be represented by (11).

The second partial derivatives of (11) are

$$
F_{m m}(x, y, z)=\left(-\frac{1}{\sigma_{m}^{2}}+\frac{m^{2}}{\sigma_{m}^{4}}\right) F_{\text {ellipsoid }}+N_{m m}(x, y, z)
$$

and

$$
F_{m n}(x, y, z)=\left(\frac{m n}{\sigma_{m}^{2} \sigma_{n}^{2}}\right) F_{\text {ellipsoid }}+N_{m n}(x, y, z),
$$

where $m, n \in\{x, y, z\}$. The noise terms $C+N(x, y, z)$ in (11) will diminish faster than the signal through the differential operation by two well-known processes. The high frequency noise will be minimized by the smoothing process of derivative estimations. The differential operator will remove low frequency noise such as the constant and ramp components. Accordingly, noise terms in the second derivatives will be relatively insignificant and can be neglected. In addition, (13) and the second term of (12) can be omitted if point $(x, y, z)$ is close to the origin where $m^{2} / \sigma_{m}^{4}$ and $m n / \sigma_{m}^{2} \sigma_{n}^{2}$ are small.

Multiply the 3D Hessian matrix $\mathbf{H}$ by unit vectors $\mathbf{v}_{m}$ $\left(\mathrm{m} \in\{x, y, z\}, \mathbf{v}_{x}=(1,0,0)^{T}, \mathbf{v}_{y}=(0,1,0)^{T}, \mathbf{v}_{z}=(0,0,1)^{T}\right)$,

$$
\begin{aligned}
& {\left[\begin{array}{lll}
F_{x x} & F_{x y} & F_{x z} \\
F_{y x} & F_{y y} & F_{y z} \\
F_{z x} & F_{z y} & F_{z z}
\end{array}\right] \mathbf{v}_{m}} \\
& \approx F_{\text {ellipsoid }}\left[\begin{array}{ccc}
-\frac{1}{\sigma_{x}^{2}}+\frac{x^{2}}{\sigma_{x}^{4}} & \frac{x y}{\sigma_{x}^{2} \sigma_{y}^{2}} & \frac{x z}{\sigma_{x}^{2} \sigma_{z}^{2}} \\
\frac{x y}{\sigma_{x}^{2} \sigma_{y}^{2}} & -\frac{1}{\sigma_{y}^{2}}+\frac{y^{2}}{\sigma_{y}^{4}} & \frac{y z}{\sigma_{y}^{2} \sigma_{z}^{2}} \\
\frac{x z}{\sigma_{x}^{2} \sigma_{z}^{2}} & \frac{y z}{\sigma_{y}^{2} \sigma_{z}^{2}} & -\frac{1}{\sigma_{z}^{2}}+\frac{z^{2}}{\sigma_{z}^{4}}
\end{array}\right] \mathbf{v}_{m} \\
& \approx F_{\text {ellipsoid }}\left[\begin{array}{ccc}
-\frac{1}{\sigma_{x}^{2}} & 0 & 0 \\
0 & -\frac{1}{\sigma_{y}^{2}} & 0 \\
0 & 0 & -\frac{1}{\sigma_{z}^{2}}
\end{array}\right] \mathbf{v}_{m} \\
& =-\frac{1}{\sigma_{m}^{2}} F_{\text {ellipsoid }} \mathbf{v}_{m} \\
& =\lambda_{m}(x, y, z) \mathbf{v}_{m} .
\end{aligned}
$$

By definition, the scalar function $\lambda_{m}$ is an eigenvalue of the Hessian matrix $\mathbf{H}$ and $\mathbf{v}_{m}$ is the corresponding eigenvector. Observed from (11) and (14), the eigenvalue $\lambda_{m}$ of the Hessian matrix derived from the Gaussian model $F$ is simply an approximation to the model scaled by a constant $-1 / \sigma_{m}^{2}$.

Based on these quantative meanings of the eigenvalues of the Hessian from the Gaussian model, we propose a simple procedure for segmenting LSCM data by thresholding on the images of eigenvalues. Let $\lambda_{1}, \lambda_{2}$, and $\lambda_{3}$ $\left(\lambda_{1} \geq \lambda_{2} \geq \lambda_{3}\right)$ be the eigenvalues of $\mathbf{H}$. The sets of sheet, line, and blob structures are defined as

$$
\begin{aligned}
& \Sigma_{\text {sheet }}\left(\sigma ; \tau_{3}\right)=\left\{\nu_{i} \mid \lambda_{3}\left(\nu_{i}\right) \leq \tau_{3}<0\right\}, \\
& \Sigma_{\text {line }}\left(\sigma ; \tau_{2}\right)=\left\{\nu_{i} \mid \lambda_{2}\left(\nu_{i}\right) \leq \tau_{2}<0\right\}, \\
& \Sigma_{\text {blob }}\left(\sigma ; \tau_{1}\right)=\left\{\nu_{i} \mid \lambda_{1}\left(\nu_{i}\right) \leq \tau_{1}<0\right\},
\end{aligned}
$$

where $\sigma$ is the standard deviation of the Gaussian filters, $\nu_{i}=(x, y, z)$ a volume element, and $\tau_{i}$ the threshold. For

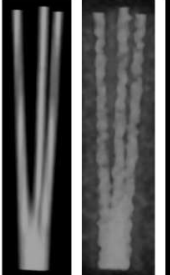

(a)

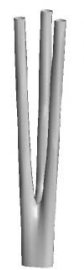

(h)

(b)

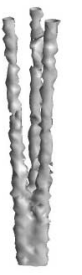

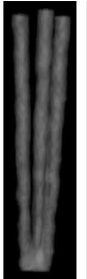
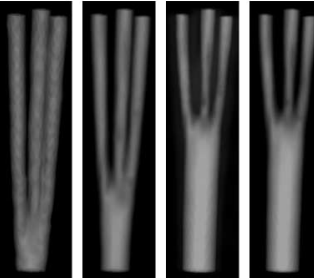

(c)

(d)

$(e)$

(f)

(g)
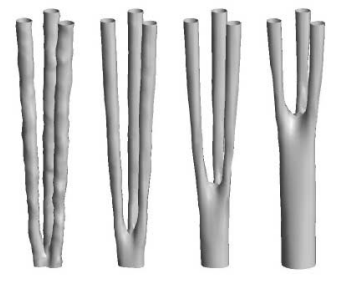

Fig. 3. A linear structure of three Gaussian lines with standard deviation $\sigma$ equal to 1.6. The distance between any lines is decreased from $5 \sigma$ at the top to $2.2 \sigma$ at the bottom. (a)-(g) Volume rendered images: (a) The noise-free data, (b) $40 \mathrm{~dB}$ Gaussian noise added, (c) $S_{\text {line }}(\sigma=1)$, (d) $S_{\text {line }}(\sigma=\sqrt{2})$, (e) $S_{\text {line }}(\sigma=2)$, (f) $S_{\text {line }}(\sigma=2 \sqrt{2})$, and (g) $M_{\text {line. }}$. (h)-(n) Surface rendered images corresponding to (a)-(g).

comparison, (15), (16), and (17) are just simplified versions of (3), (4), and (5) with $\gamma=\omega\left(\lambda_{s} ; \lambda_{t}\right)=1$.

Although (3), (4), and (5) are able to distinguish one shape from the other, they are not mutually exclusive. Take (4) and (5), for example. The only difference between them is the weight function of $\lambda_{1}$ and $\lambda_{2}$. It is easy to depict that the function curves of $\omega\left(\lambda_{1} ; \lambda_{2}\right)$ and $\psi\left(\lambda_{1} ; \lambda_{2}\right)$ intersect each other at some point between $0<\lambda_{1} / \lambda_{2} \leq 1$, where $\lambda_{2} \leq \lambda_{1}<0$. While $\Sigma_{\text {sheet }}\left(\tau_{3}\right), \Sigma_{\text {line }}\left(\tau_{2}\right)$, and $\Sigma_{\text {blob }}\left(\tau_{1}\right)$ are neither shape-selective nor mutually exclusive, however, there exist inclusive relationships between them. In fact,

$$
\Sigma_{\text {blob }} \subset \Sigma_{\text {line }} \subset \Sigma_{\text {sheet }}
$$

for $\tau_{1}=\tau_{2}=\tau_{3}$. With the inclusive property, it is more intuitive to visualize the transition perceptively from one shape category to the other for diffused structures such as cellular organelles.

\subsection{Line Filtering Evaluation}

A line filter with a single scale can give a high response in only a narrow range around the chosen scale. Therefore, it is impractical to detect line structures with various widths. Instead, multiple, normalized filters with different scales are generally applied to the same data set and the maximum response is collected. This multiscale approach can be summarized by

$$
M_{\text {line }}=\max S_{\text {line }}\left(\sigma_{i}\right) .
$$

While the width response curve for (19) can be perfectly uniform if continuous variation values are used, it is reported in [27] that the response curve deviates less than 3 percent between $\sigma_{i}=1$ and $\sigma_{i}=2 \sqrt{2}$ using a set of discrete values $\sigma_{i} \in\{1, \sqrt{2}, 2,2 \sqrt{2}\}$. This range is appropriate for most applications.

Next, we examine the multiscale filtering approach with a linear structure which consists of three closely placed lines. Fig. 3a illustrates a linear structure of three Gaussian 


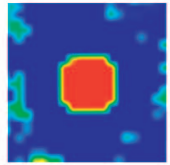

(a)

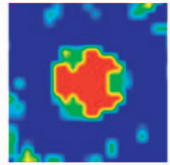

(b)

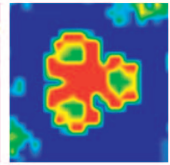

(c)

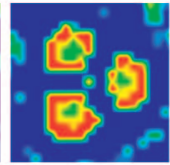

(d)

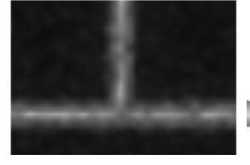

(a)

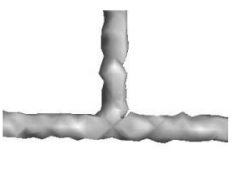

(b)
Fig. 4. Four cross sections of the resultant image of $M_{\text {line }}$ (Fig. $3 \mathrm{~g}$ ) at $\mathrm{z}$ equal to (a) 0 (bottom), (b) 0.34 , (c) 0.74 , and (d) 1 (top) colored by blue, green, yellow, and red based on the maximum responses by $\sigma=1, \sqrt{2}$, 2 , and $2 \sqrt{2}$, respectively.

line functions which have standard deviation $\sigma$ equal to 1.6. The distance between any two lines is decreased from $5 \sigma$ at the top to $2.2 \sigma$ at the bottom. The test data is added with $40 \mathrm{~dB}$ Gaussian noise as shown in Fig. 3b. (We use the definition $\mathrm{SNR}=20 \log \frac{S}{\sigma^{2}}$ with $\sigma_{\text {noise }}^{2}$, the variance of the Gaussian noise, and $\mathrm{S}$ the maximum signal strength.) The multiscale filtering method described in [27], summarized by (4) and (19) with $\alpha=0.25$ and $\gamma=0.5$, is then applied to the noisy data (Fig. 3b). The single-scale line filtering responses $S_{\text {line }}$ for $\sigma=1, \sqrt{2}, 2,2 \sqrt{2}$ and the multiscale response $M_{\text {line }}$ are shown in Figs. 3c, 3d, 3e, 3f, and $3 g$ by volume rendering. The isosurface rendered images are shown in Figs. 3h, 3i, 3j, 3k, 31, 3m, and 3n, correspondingly. The resultant images indicate that, the larger $\sigma$ is, the less likely the filter would be able to distinguish closely positioned line structures.

Fig. 4 displays four cross sections of the resultant image of $M_{\text {line }}$ at $\mathrm{z}$ equal to 0 (bottom), 0.34, 0.74, and 1 (top). The coloring scheme for Fig. 4 is based on the value $\sigma$ generating the maximum response $S_{\text {line }}(\sigma)$. Voxels with maximum response at $\sigma=1, \sqrt{2}, 2$, and $2 \sqrt{2}$ are assigned with color blue, green, yellow, and red, respectively. Fig. 4a shows that the filter with $\sigma=2 \sqrt{2}$ (colored red) dominates the multiple responses as the lines get closer and, therefore, makes the multiscale approach less sensitive to closely,positioned line structures.

Alternatively, we apply the simplified line segmentation scheme $\Sigma_{\text {line }}$ (16), to the same data set (Fig. 3b) by six single-scale Gaussian filters with standard deviations $\sigma=1$, $\sqrt{2}, 1.6,1.8,2$, and $2 \sqrt{2}$. Fig. 5 illustrates the results by thresholding on eigenvalue $\lambda_{2}$. It shows the same trend as the implementation of $S_{\text {line }}$ that, the larger $\sigma$ is, the less sensitive the line filter $\Sigma_{\text {line }}$. It also shows that there is a high degree of resemblance between the results of $\Sigma_{\text {line }}$ and $S_{\text {line }}$ for $\sigma=1, \sqrt{2}, 2$, and $2 \sqrt{2}$.

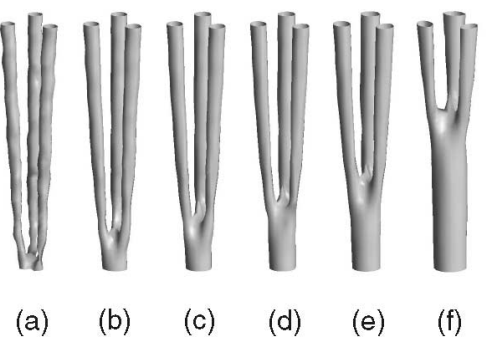

Fig. 5. Surface rendered images of simplified line segmentation scheme $\Sigma_{\text {line }}(\sigma)$ applied to the data shown in Fig. 3b with $\sigma$ equal to (a) 1, (b) $\sqrt{2}$, (c) 1.6 , (d) 1.8, (e) 2 , and (f) $2 \sqrt{2}$.

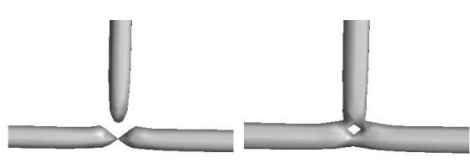

(c)

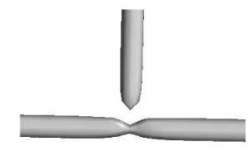

(f) (d)

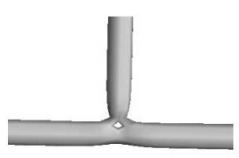

(g)

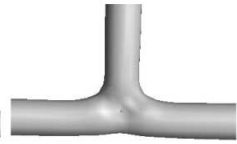

(e)

(h)

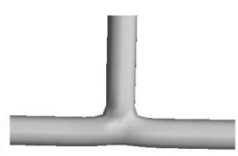

Fig. 6. A branch model created with standard deviation $\sigma=1.6$ and $40 \mathrm{~dB}$ noise added. (a) Volume rendered image. (b) Surface rendered image. (c), (d), and (e) Surface rendered images of $M_{\text {line }}$ at high, medium, and low thresholds. (f), (g), and (h) Surface rendered images of $\Sigma_{\text {line }}(\sigma=1.6)$ at high, medium, and low thresholds.

Last, we test methods $M_{\text {line }}$ and $\Sigma_{\text {line }}(\sigma=1.6)$ with a branch model which is created with $\sigma$ equal to 1.6 and $40 \mathrm{~dB}$ Gaussian noise added. The results are shown in Fig. 6. As the ellipsoidal Gaussian model is no longer accurate at the intersection, the filtering response becomes smaller at the intersection and could cause disconnection (Figs. $6 \mathrm{c}$ and $6 \mathrm{f}$ ), or a hole (Figs. $6 \mathrm{~d}$ and $6 \mathrm{~g}$ ) with a high threshold. The problem can be erased by lowering the thresholding value. However, this will cause thickening effects as in Figs. 6e and $6 \mathrm{~h}$. Fig. 6e requires a lower thresholding value to recover the hole at the branching point and appears thicker than Fig. 6h. This is because the size of the hole is proportional to $\sigma$ and $M_{\text {line }}$ is dominated by large $\sigma$.

\section{Results}

First, the Hessian-based, normalized line filter $S_{\text {line }}(\sigma)$ with scale $\sigma=1, \sqrt{2}, 2$, and $2 \sqrt{2}$ is applied to a LSCM mouse egg data set (Fig. 1a). The line filtering responses are shown in Figs. $7 \mathrm{a}, 7 \mathrm{~b}, 7 \mathrm{c}$, and $7 \mathrm{~d}$ by volume rendering. The volume rendered images of eigenvalue $\lambda_{2}$ (which defines the simplified line filter $\left.\Sigma_{\text {line }}(\sigma)\right)$ acquired by the same set of scales are shown in Figs. 7e, 7f, 7g, and 7h. Both of them show that the sensitivity of detecting closely positioned fibers decreases as larger $\sigma$ is applied. This trend is consistent with the results obtained from the synthesized linear structure evaluation in Section 3.2. However, the mouse egg spindle image is much noisier where filter responses by small $\sigma$ appear bumpy and fragmentary. Images of eigenvalue $\lambda_{2}$ computed by Gaussian filters with $\sigma=1.6$ and 1.8 are shown in Figs. $7 \mathrm{i}$ and $7 \mathrm{j}$ for comparison. They appear smoother with a slight loss of sensitivity.

Figs. 7a, 7b, 7c, and 7d and Figs. 7e, 7f, 7g, and 7h share a great degree of resemblance except that the latter ones contain some "thorns" at the right pole of the spindle and some fibers. The isosurface rendered images are shown in Fig. 8. The thorns are more visible in Figs. $8 \mathrm{f}$ and 8g, compared to Figs. $8 \mathrm{~b}$ and $8 \mathrm{c}$. These short thorn structures appear like blobs and are more likely to be removed by filter $S_{\text {line }}$. Although the 


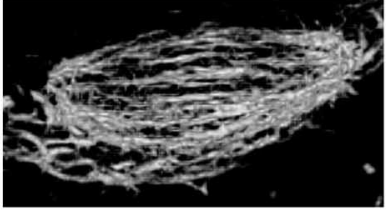

(a)

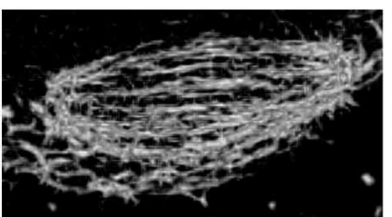

(e)

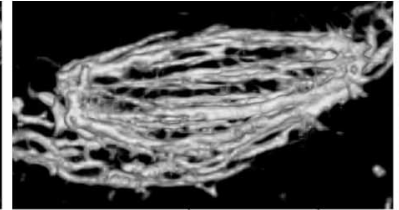

(b)

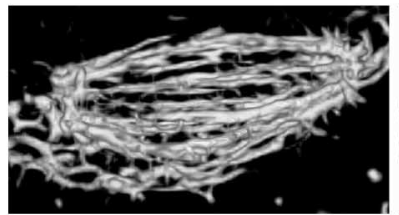

(f)

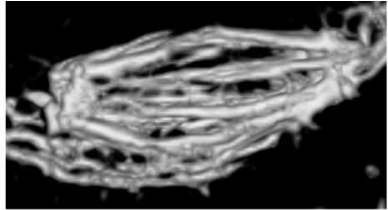

(c)

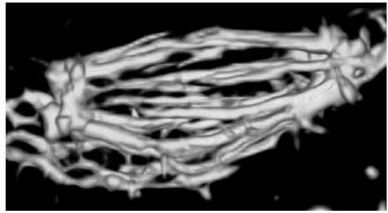

(g)

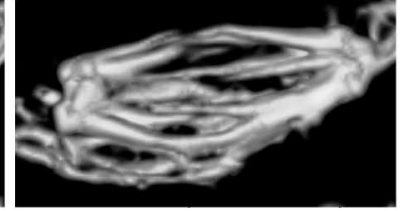

(d)

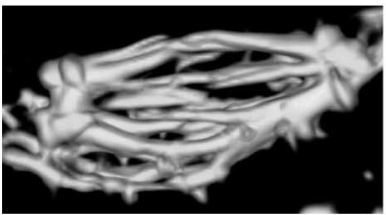

(h)

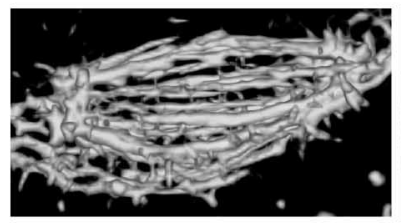

(i)

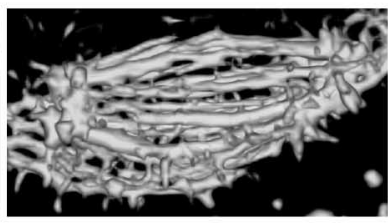

(j)

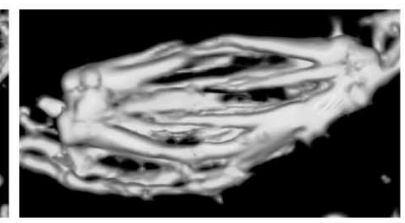

(k)

Fig. 7. Volume rendering of mouse egg spindle fibers from LSCM. (a) $S_{\text {line }}(\sigma=1)$. (b) $S_{\text {line }}(\sigma=\sqrt{2})$. (c) $S_{\text {line }}(\sigma=2)$. (d) $S_{\text {line }}(\sigma=2 \sqrt{2})$. (e) $\Sigma_{\text {line }}(\sigma=1)$. (f) $\Sigma_{\text {line }}(\sigma=\sqrt{2})$. (g) $\Sigma_{\text {line }}(\sigma=2)$. (h) $\Sigma_{\text {line }}(\sigma=2 \sqrt{2})$. (i) $\Sigma_{\text {line }}(\sigma=1.6)$. (j) $\Sigma_{\text {line }}(\sigma=1.8)$. (k) $M_{\text {line }}$.

scientific significance of polar thorns in a mouse egg spindle remains to be explored and studied, the simplified shape filter $\Sigma_{\text {line }}(\sigma)$ has the inclusive property that short line structures can be observed and quantitatively related to the ellipsoidal model's standard deviations. Therefore, it is a better filter for cellular structure visualization.

The maximum response $M_{\text {line }}$ is shown in Figs. $7 \mathrm{k}$ and $8 \mathrm{k}$. Several cross sections of the spindle structure are displayed in Figs. 9a, 9b, 9c, 9d, 9e, 9f, and 9g. The scale which generates the maximum response at each voxel on these cross sections is illustrated in Figs. 9h, 9i, 9j, 9k, 9l, 9m, and 9 n. The maximum responses by $\sigma=1, \sqrt{2}, 2$, or $2 \sqrt{2}$ are colored by blue, green, yellow, or red, respectively. Again, we observe that the large scale tends to dominate the response as the spindle fibers get nearer to both poles. The results are all consistent with the ellipsoid modeling and synthetic data evaluation in Section 3.

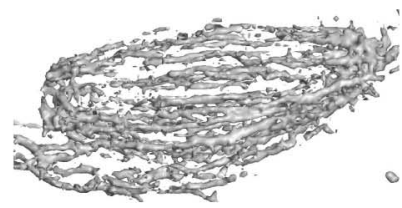

(a)

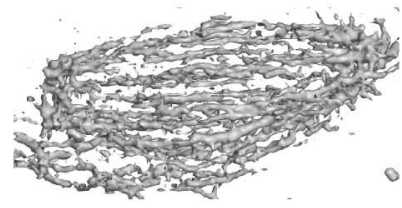

(e)

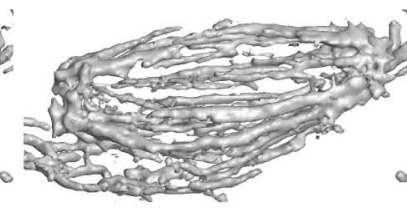

(b)

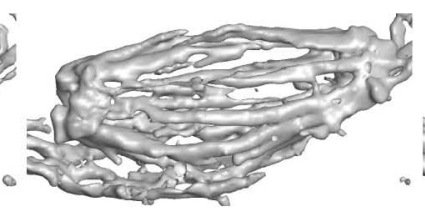

(c)

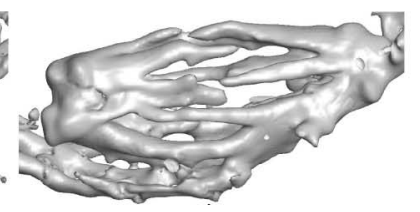

(d)

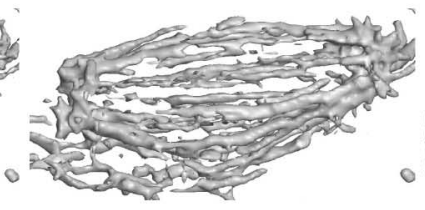

(f)

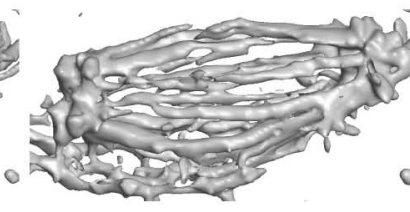

(g)

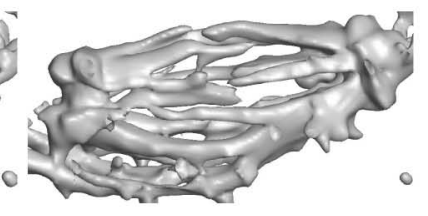

(h)

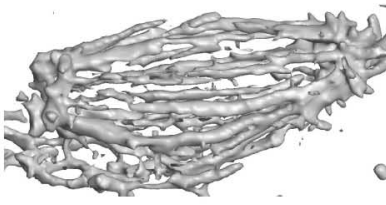

(i)

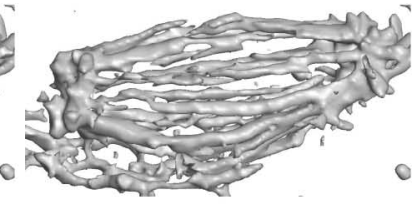

(j)

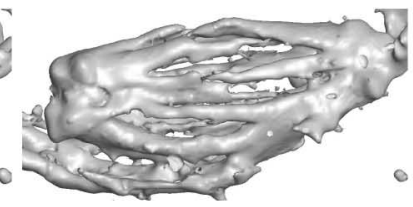

(k)

Fig. 8. Surface rendering of mouse egg spindle fibers from LSCM. (a) $S_{\text {line }}(\sigma=1)$. (b) $S_{\text {line }}(\sigma=\sqrt{2})$. (c) $S_{\text {line }}(\sigma=2)$. (d) $S_{\text {line }}(\sigma=2 \sqrt{2})$. (e) $\Sigma_{\text {line }}(\sigma=1)$. (f) $\Sigma_{\text {line }}(\sigma=\sqrt{2})$. (g) $\Sigma_{\text {line }}(\sigma=2)$. (h) $\Sigma_{\text {line }}(\sigma=2 \sqrt{2})$. (i) $\Sigma_{\text {line }}(\sigma=1.6)$. (j) $\Sigma_{\text {line }}(\sigma=1.8)$. (k) $M_{\text {line }}$. 


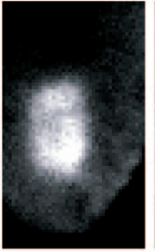

(a)

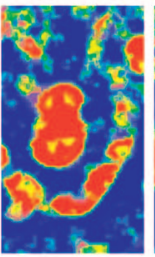

(h)

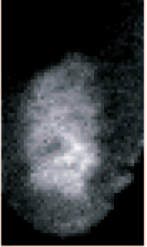

(b)

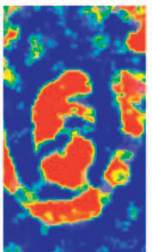

(i)

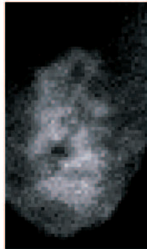

(c)

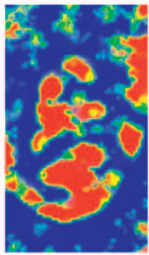

(j)

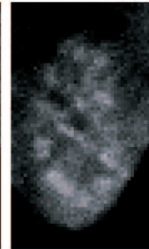

(d)

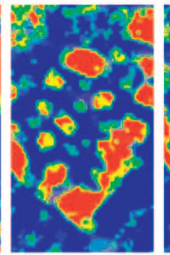

(k)

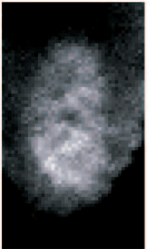

(e)

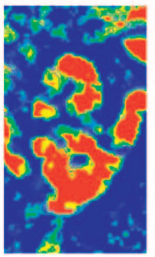

(I)

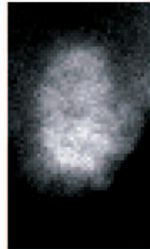

(f)

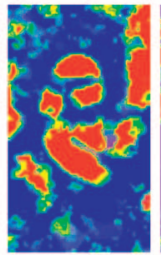

(m)

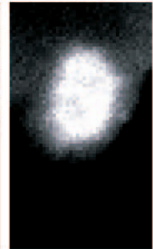

(g)

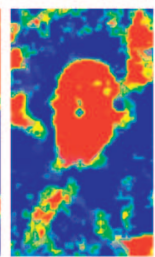

(n)

Fig. 9. (a), (b), (c), (d), (e), (f), and (g) Cross sections of the spindle structure (LSCM data). (h), (i), (j) (k), (l), (m), and (n) Cross sections of $M_{\text {line }}$ colored by blue, green, yellow, and red based on the maximum responses by $\sigma=1, \sqrt{2}$, 2 , and $2 \sqrt{2}$, respectively.

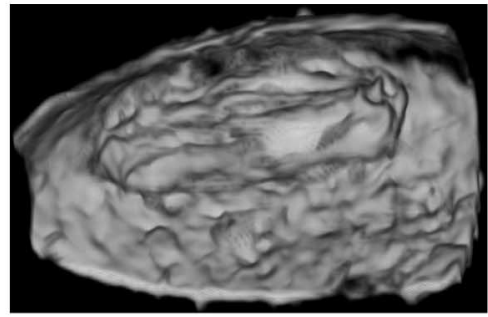

(a)

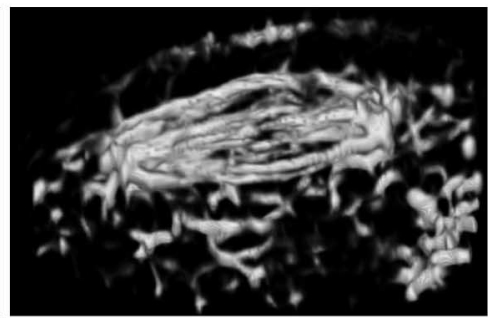

(b)

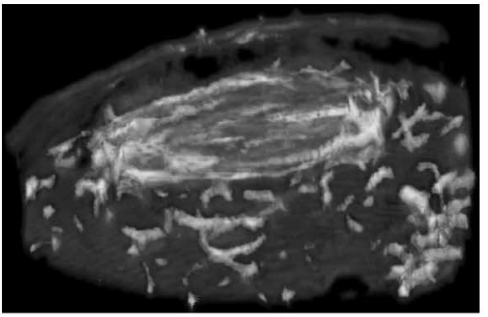

(c)

Fig. 10. Plasma membrane and spindle fiber images generated by simplified segmentation schemes $\Sigma_{\text {sheet }}$ and $\Sigma_{\text {line }}$ with scale $\sigma=1.8$. (a) $\Sigma_{\text {sheet }}(\sigma=1.8)$. (b) $\Sigma_{\text {line }}(\sigma=1.8)$. (c) Composite image of (a) and (b).

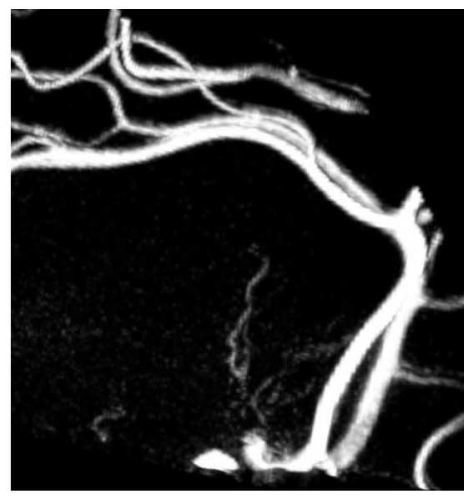

(a)

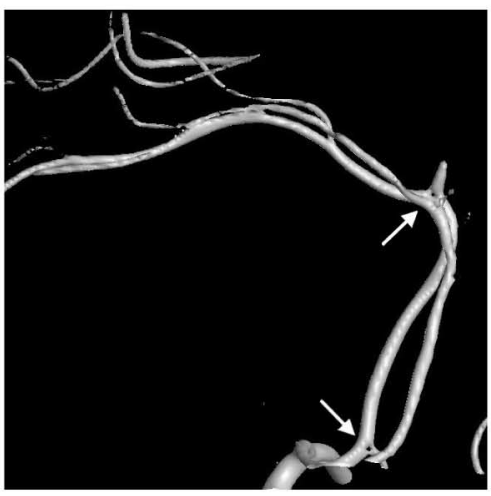

(b)

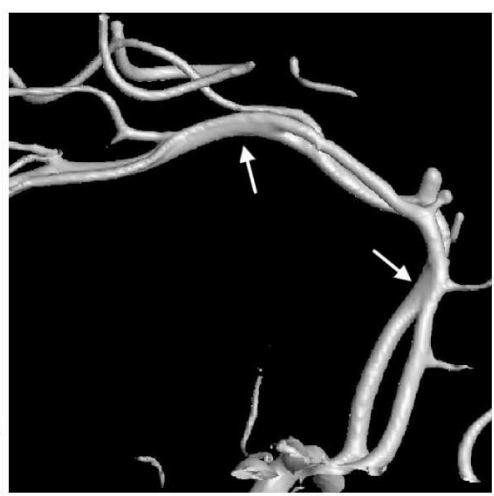

(c)

Fig. 11. A blood vessel data set. (a) Rendered by maximum intensity projection. (b) Surface rendered image of $M_{\text {line }}$ at a high threshold with holes at branches. (c) Surface rendered image of $M_{\text {line }}$ at a lower threshold with fused vessels.

Next, we apply the new, simplified sheet filter $\Sigma_{\text {sheet }}(\sigma=1.8)$ and line filter $\Sigma_{\text {line }}(\sigma=1.8)$ to the same mouse egg spindle with partial plasma membrane nearby. $\Sigma_{\text {sheet }}$ and $\Sigma_{\text {line }}$ are simply the images of eigenvalues $\lambda_{3}$ and $\lambda_{2}$ by thresholding. These terms are used interchangeably here to name the processed images. Fig. 10a shows the image of $\Sigma_{\text {sheet }}$ by volume rendering with a step opacity function of $\lambda_{3}$. Besides sheet-like membrane, Fig. 10a shows that $\Sigma_{\text {sheet }}$ also encloses the whole spindle. This corresponds with the inclusive property, $\Sigma_{\text {blob }} \subset \Sigma_{\text {line }} \subset \Sigma_{\text {sheet }}$, discussed in Section 3.1. To extract line structure, the same rendering procedure is applied to $\lambda_{2}$ using an adjusted opacity function and a different color table. Fig. 10b shows the rendered image of $\Sigma_{\text {line }}$. Similarly, image $\Sigma_{b l o b}$ can be derived this way. However, the blob structures are not targeted by this LSCM module and, therefore, omitted in this experiment. Fig. 10c shows the combined image of $\Sigma_{\text {sheet }}$ and $\Sigma_{\text {line }}$.

Last, we examine a blood vessel data set with the multiscale approach. Fig. 11a is a blood vessel image rendered by maximum intensity projection. Figs. $11 \mathrm{~b}$ and 11c are surface rendered images of the maximum response 
$M_{\text {line }}$ by thresholding on two different values. At a high threshold, the hole problem appears at two branching areas shown in Fig. 11b. As the thresholding value is lowered to recover small holes at the branching points, the effects of line fusion appear in Fig. 11c.

\section{Conclusions}

This paper presents a case where the multiscale approach falls short. A linear structure of closely positioned fibers is used to evaluate the sensitivity of normalized line filtering and the maximum response. We find that sensitivity in detecting closely placed lines decreases as the standard deviation (filter scale) increases. We also find that the maximum response at closely placed lines tends to be dominated by large scale filters. Therefore, the multiscale approach is unsuitable to detect spindle fibers. In addition, we simplify existing shape filters through an ellipsoidal Gaussian model. We find that the eigenvalues of the Hessian matrix are quantitatively associated with the standard deviations of the ellipsoid model. The sheet, line, and blob filters are replaced by the eigenvalues of the Hessian matrix instead. Our new methods are implemented with a singlescale filter, balanced between resistance to noise and sensitivity. They are able to detect cellular fibers and plasma membranes as well. Although they are unable to discriminate line or blob structures from a sheet structure, they have an inclusive property $\Sigma_{\text {blob }} \subset \Sigma_{\text {line }} \subset \Sigma_{\text {sheet }}$. With the inclusive property, it is more intuitive to perceptively visualize the transition from one shape category to the other for diffused structures such as cellular organelles. Further, without suppressing short fibers as blobs, we are able to visualize a polar thorn phenomenon.

\section{ACKNOWLEDGMENTS}

This work was done while A. Huang was a graduate student at Arizona State University (ASU). It was supported by the US National Science Foundation (NSF IIS-9980166 and ACI-0083609), the US Office of Naval Research (N00014-00-1-0281), and the US Defense Advanced Research Projects Agency (MDA972-00-1-0027). The authors would like to thank the PRISM Laboratory, the W.M. Keck BioImaging Laboratory, and the Cell Biology Laboratory at ASU for providing the laboratory and computing resources. Retinal vessel image comes from the Biomedical Image Archive, University of Bristol, United Kingdom, and is provided by Professor Hirst at Princess Alexandra Hospital, Brisbane. Brain vessel data comes from www.volvis.org and is provided by Philips Research, Hamburg, Germany. The volume rendered images are created by VolView 2.0 provided by Kitware Inc., Clifton Park, New York.

\section{REFERENCES}

[1] S. Aylward, S.M. Pizer, E. Bullitt, and D. Eberly, "Intensity Ridge and Widths for 3D Object Segmentation and Description," Proc. IEEE Workshop Math. Methods in Biomedical Image Analysis, pp. 131138, 1996.

[2] D.P. Baluch and D.G. Capco, "Cellular Scaffolds in Mammalian Eggs," Frontiers in Bioscience, vol. 7, pp. 1653-1661, July 2002.

[3] P.J. Basser, J. Mattiello, and D. LeBihan, "MR Diffusion Tensor Spectroscopy and Imaging," Biophyisical J., vol. 66, pp. 259-267, 1994.

[4] J.-D. Boissonnat, "Shape Reconstruction from Planar CrossSections," Computer Vision, Graphics, and Image Processing, vol. 44, pp. 1-29, 1988.
[5] S. Chaudhuri, S. Chatterjee, N. Katz, M. Nelson, and M. Goldbaum, "Detection of Blood Vessels in Retinal Images Using Two-Dimensional Matched Filters," IEEE Trans. Medical Imaging, vol. 8, no. 3, pp. 263-269, Sept. 1989.

[6] R.A. Drebin, L. Carpenter, and P. Hanrahan, "Volume Rendering," Computer Graphics (Proc. SIGGRAPH '88), vol. 22, pp. 65-73, 1988.

[7] H. Edelsbrunner and E.P. Muecke, "Three-Dimensional Alpha Shape," Proc. Workshop Volume Visualization, pp. 75-105, Oct. 1992.

[8] G. Farin, "Shape," Math. Unlimited-2001 and Beyond, E. Engquist and W. Schmid, eds., pp. 463-466, Springer-Verlag, 2001.

[9] A.F. Frangi, W.J. Niessen, K.L. Vincken, and M.A. Viergever, "Multiscale Vessel Enhancement Filtering," Medical Image Computing and Computer-Assisted Intervention (MICCAI '98), pp. 130-137, 1998.

[10] L. Gang, O. Chutatape, and S.M. Krishnan, "Detection and Measurement of Retinal Vessels in Fundus Images Using Amplitude Modified Second-Order Gaussian Filter," IEEE Trans. Biomedical Eng., vol. 49, no. 2, pp. 168-172, Feb. 2002.

[11] H. Hoppe, T. DeRose, T. Duchamp, J. McDonald, and W. Stuetzle, "Surface Reconstruction from Unorganized Points," Proc. SIGGRAPH '92, pp. 71-78, July 1992.

[12] J. Hu, A. Razdan, G. Nielson, G. Farin, D. Baluch, and D. Capco, "Volumetric Segmentation Using Weibull E-SD Fields," IEEE Trans. Visualization and Computer Graphics, vol. 9, no. 3, pp. 320328, July-Sept. 2003.

[13] A. Huang, "Three-Dimensional Biomedical Image Segmentation and Visulaization: A Shape-Based Approach," PhD Dissertation, Arizona State Univ., 2003.

[14] A. Huang and G.M. Nielson, "Surface Approximation to Point Cloud Data Using Volume Modeling," Data Visualization, F. Post, G. Nielson, and G.P. Bonneau, eds., pp. 333-343, Elsevier, 2002.

[15] A. Huang, G. Nielson, A. Razdan, G. Farin, D. Capco, and P. Baluch, "Line and Net Pattern Segmentation Using Shape Modeling," Proc. SPIE Electronic Imaging Symp. Visualization and Data Analysis, 2003.

[16] A. Huang, R.M. Summers, and A.K. Hara, "Surface Curvature Estimation for Automatic Colonic Polyp Detection," Medical Imaging 2005, pp. 393-402, 2005.

[17] M. Kass, A. Witkin, and D. Terzopoulos, "Snakes: Active Contour Models," Int'l J. Computer Vision, pp. 321-331, 1988.

[18] E. Kepple, "Approximating Complex Surfaces by Triangulation of Contour Lines," IBM J. Research and Development, vol. 19, pp. 2-11, 1975.

[19] G. Kindlmann, R. Whitaker, T. Tasdizen, and T. Möller, "Curvature-Based Transfer Functions for Direct Volume Rendering: Methods and Applications," Proc. IEEE Visualization Conf. 2003, pp. 513-520, 2003.

[20] M. Levoy, "Volume Rendering: Display of Surfaces from Volume Data," IEEE Computer Graphics and Applications, pp. 29-36, 1988.

[21] W.E. Lorensen and H.E. Cline, "Marching Cubes: A High Resolution 3D Surface Construction Algorithm," Computer Graphics, vol. 21, no. 4, pp. 163-169, July 1987.

[22] L.M. Lorigo, O. Faugeras, W.E.L. Grimson, R. Keriven, R. Kikinis, and C.-F. Westin, "Co-Dimension 2 Geodesic Active Contours for MRA Segmentation," Proc. Int'l Conf. Information Processing in Medical Imaging (IPMI '99), pp. 126-133, 1999.

[23] R. Malladi, J.A. Sethian, and B.C. Vemuri, "Shape Modeling with Front Propagation: A Level Set Approach," IEEE Trans. Pattern Analysis and Machine Intelligence, vol. 17, no. 2, pp. 158-175, Feb. 1995.

[24] G.M. Nielson, A. Huang, and S. Sylvester, "Approximation Normals for Marching Cubes Applied to Locally Supported Isosurfaces," Proc. IEEE Visualization Conf. 2002, pp. 459-466, Oct. 2002.

[25] A. Razdan, K. Patel, G. Farin, and D.G. Capco, "Visualization of Multicolor LCM Data Set," Computers and Graphics, vol. 25, no. 3, pp. 371-382, 2001.

[26] Y. Sato, S. Nakajima, N. Shiraga, H. Atsumi, S. Yoshida, T. Koller, G. Gerig, and R. Kikinis, "Three-Dimensional Multiscale Line Filter for Segmentation and Visualization of Curvilinear Structures in Medical Images," Medical Image Analysis, vol. 2, no. 2, pp. 143-168, 1998

[27] Y. Sato, C.F. Westin, A. Bhalerao, S. Nakajima, N. Shiraga, S. Tamura, and R. Kikinis, "Tissue Classification Based on 3D Local Intensity Structures for Volume Rendering," IEEE Trans. Visualization and Computer Graphics, vol. 6, no. 2, pp. 160-180, Apr.-June 2000. 
[28] A.W. Simon, D. Van Alfen, A. Razdan, G. Farin, M. Bae, and J. Rowe, "3D Modeling for Analysis and Archiving of Ceramic Vessel Morphology: A Case Study from the American Southwest," Proc. 33rd Int'l Symp. Archaeometry, pp. 257-263, 2002.

[29] G. Stylianou and G. Farin, "Crest Lines for Segmentation and Flattening," IEEE Trans. Visualization and Computer Graphics, vol. 10, pp. 536-544, 2004.

[30] R.M. Summers, C.F. Beaulieu, L.M. Pusanik, J.D. Malley, R.B. Jeffrey Jr., D.I. Glazer, and S. Napel, "An Automated Polyp Detector for CT Colonography-Feasibility Study," Radiology, vol. 216, pp. 284-290, 2000.

[31] H.J. Tiziani and H.M. Uhde, "Three Dimensional Image Sensing with Chromatic Confocal Microscopy," Applied Optics, vol. 33, pp. 1838-1843, 1994.

[32] H. Yoshida, Y. Masutani, P. MacEneaney, D.T. Rubin, and A.H. Dachman, "Computerized Detection of Colonic Polyps at CT Colonography on the Basis of Volumetric Features: Pilot Study," Radiology, vol. 222, pp. 327-336, 2002.

[33] L. Zhukov and A.H. Barr, "Heart-Muscle Fiber Reconstruction from Diffusion Tensor MRI," Proc. IEEE Visualization Conf., 2003.

[34] L. Zhukov and A.H. Barr, "Oriented Tensor Reconstruction: Tracing Neural Pathways from Diffusion Tensor MRI," Proc. IEEE Visualization Conf., pp. 387-394, 2002.

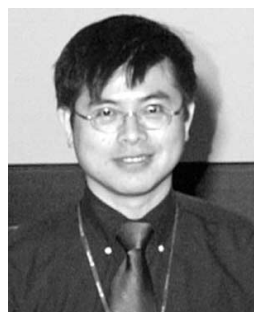

Adam Huang received the BS and MS degrees in electrical engineering from National Tsing Hua University, Hsinchu, Taiwan, in 1991, and Polytechnic University, Brooklyn, New York, in 1996, respectively. He also received the MS and $\mathrm{PhD}$ degrees in computer science from Arizona State University in 1999 and 2003. He is currently a visiting postdoctoral fellow in the Diagnostic Radiology Department of Warren Grant Magnuson Clinical Center at the National Institutes of Health, where he conducts research in computer-aided diagnostic CT colonography at the Virtual Endoscopy and ComputerAided Diagnosis Lab of Dr. Ron Summers. His research interests include biomedical image processing, computer-aided geometric design, computer graphics, and scientific visualization.

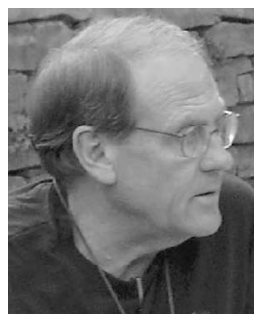

Gregory M. Nielson received the $\mathrm{PhD}$ degree from the University of Utah. He is a professor of computer science and affiliate professor of mathematics at Arizona State University where he teaches and does research in the areas of computer graphics, computer-aided geometric design, and scientific visualization. He has lectured and published widely on the topics of curve and surface representation and design, interactive computer graphics, scattered data modeling, and the analysis and visualization of multivariate data. He has edited several books and authored more than 100 scientific articles. $\mathrm{He}$ has collaborated with several institutions including NASA, Xerox, General Motors, and Lawrence Livermore National Laboratory. He has been on the editorial boards of the ACM Transactions on Graphics, The Rocky Mountain Journal of Mathematics, IEEE Computer Graphics and Applications, Visualization, and the Computer Animation Journal. He is currently on the editorial board of Computer Aided Geometric Design and the editorial advisory board of the IEEE Transactions on Visualization and Computer Graphics. He is one of the founders and members of the steering committee of the IEEE sponsored conference, Visualization. He has previously chaired and is currently a director of the IEEE Computer Society Technical Committee on Computer Graphics. $\mathrm{He}$ is the recipient of an IEEE Meritorious Service Award, an IEEE Outstanding Contribution Award, and the John Gregory Memorial Award in Geometric Modeling. He is a senior member of the IEEE.

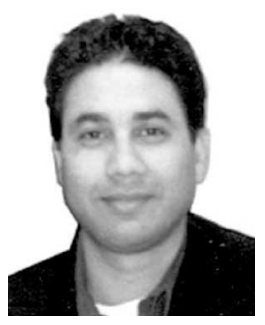

Anshuman Razdan received the BS and MS degrees in mechanical engineering and the $\mathrm{PhD}$ degree in computer science. He is the director of PRISM: Partnership for Research In Stereo Modeling at Arizona State University. His research interests include computer-aided geometric design (CAGD) and computer graphics, NURB curves and surfaces approximation, feature segmentation for surface and volume data, and use of high bandwidth networking for scientific visualization. He is a $\mathrm{PI}$ on several US National Science Foundation grants, including a recent 2.1 M KDI grant on 3D Knowledge: Acquisition, Representation, and Analysis (3DK). $\mathrm{He}$ is a member of the IEEE.

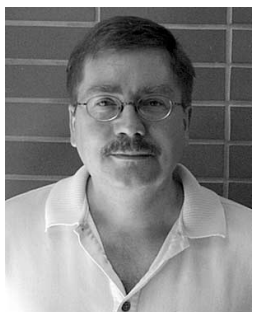

Gerald E. Farin received the $\mathrm{PhD}$ degree in mathematics from the University of Braunschweig, Germany, in 1979. He is the author of the texts Curves and Surfaces for CAGD (fifth edition), NURBS (second edition), The Geometry Toolbox, and The Essentials of CAGD. He is editor-in-chief of Computer Aided Geometric Design. His industrial experience consists of four years of CAD/CAM development at Mercedes-Benz, Stuttgart, Germany. He subsequently worked at the University of Utah (mathematics) and Arizona State University (computer science). He has taught CAGD tutorials worldwide and is the author of more than 100 research publications.

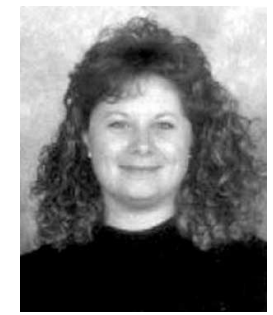

D. Page Baluch received the BA degree in biology from the University of Colorado at Colorado Springs in 1999. She is currently pursuing the $\mathrm{PhD}$ degree in biology at Arizona State University. She works as a research assistant in the Partnership for Research in Stereo Modeling (PRISM) lab and conducts her graduate studies in the Molecular and Cellular Biology lab of Dr. David Capco in the Biology Department. Her current interests are studying the cytoskeletal elements involved in the signal transduction pathways of mouse eggs before and after fertilization.

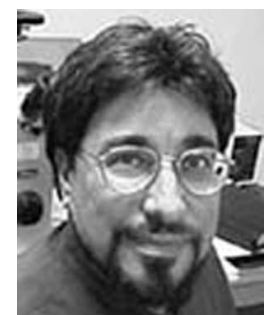

David G. Capco received the PhD degree from the University of Texas at Austin in cell and developmental biology in 1980 and underwent further training during his postdoctoral work at MIT before becoming a member of the faculty at Arizona State University in 1984. He is a professor of biology at Arizona State University. His research interests focus on the signal transduction machinery that regulate cell function with an emphasis on signaling agents which regulate the cell cycle, specifically in mammalian eggs. His research examines mechanisms to increase the efficiency of cloning of agricultural animals.

$\triangleright$ For more information on this or any other computing topic, please visit our Digital Library at www.computer.org/publications/dlib. 